\title{
A Reevaluation of the Inverse Dynamic Model for Eye Movements
}

\author{
Andrea M. Green, ${ }^{1}$ Hui Meng, ${ }^{2}$ and Dora E. Angelaki ${ }^{2}$ \\ 'Département de Physiologie, Université de Montréal, Montréal, Québec, Canada H3T 1J4, and 2Department of Anatomy and Neurobiology, Washington \\ University School of Medicine, St. Louis, Missouri 63110
}

\begin{abstract}
To construct an appropriate motor command from signals that provide a representation of desired action, the nervous system must take into account the dynamic characteristics of the motor plant to be controlled. In the oculomotor system, signals specifying desired eye velocity are thought to be transformed into motor commands by an inverse dynamic model of the eye plant that is shared for all types of eye movements and implemented by a weighted combination of eye velocity and position signals. Neurons in the prepositus hypoglossi and adjacent medial vestibular nuclei (PH-BT neurons) were traditionally thought to encode the "eye position" component of this inverse model. However, not only are PH-BT responses inconsistent with this theoretical role, but compensatory eye movement responses to translation do not show evidence for processing by a common inverse dynamic model. Prompted by these discrepancies between theoretical notions and experimental observations, we reevaluated these concepts using multiple-frequency rotational and translational head movements. Compatible with the notion of a common inverse model, we show that $\mathrm{PH}-\mathrm{BT}$ responses are unique among all premotor cell types in bearing a consistent relationship to the motor output during eye movements driven by different sensory stimuli. However, because their responses are dynamically identical to those of motoneurons, PH-BT neurons do not simply represent an internal component of the inverse model, but rather its output. They encode and distribute an estimate of the motor command, a signal critical for accurate motor execution and learning.
\end{abstract}

Key words: internal model; eye movement; vestibular; efference copy; sensorimotor; motor control

\section{Introduction}

In any motor system, the brain must compute motor commands from signals that provide a representation of desired action. The required computations often rely on internal representations of the dynamic properties of the motor plant to be controlled. Such "internal models," which constitute a general theoretical concept in motor control, may be used either to transform desired action into appropriate motor commands ("inverse model") or conversely to predict the consequences of motor commands on behavior ("forward model") (Shidara et al., 1993; Shadmehr and Mussa-Ivaldi, 1994; Miall and Wolpert, 1996; Kawato, 1999).

The neural correlates of an inverse model are perhaps best understood in the oculomotor system, where its existence was established early on because the broad bandwidth of the rotational vestibulo-ocular reflex (RVOR) implies neural compensation for the eye plant dynamics (Skavenski and Robinson, 1973). Desired eye velocity was proposed to be combined with a neurally computed eye position signal to construct a simplified (firstorder) inverse dynamic representation of the eye plant (Skavenski and Robinson, 1973; Robinson, 1981). This theoretical con-

Received Sept. 1, 2006; revised Nov. 27, 2006; accepted Dec. 20, 2006.

This work was supported by National Institute of Health Grants F32-DC05271, EY12814, and DC04260. We thank Paul Cisek, Steve Lisberger, and Eliana Klier for comments on versions of this manuscript, Kim Kocher for excellent technical assistance, and Chris Kaneko for his expert advice and for contributing custom-made electrodes. We also thank Paul May and David Dickman for assistance with histological reconstructions of recording sites.

Correspondence should be addressed to Dr. Andrea Green, Département de Physiologie, Université de Montréal, 2960 Chemin de la Tour, Room 2140, Montréal, Québec, Canada H3T 1J4. E-mail: andrea.green@umontreal.ca. DOI:10.1523/JNEUROSCI.3822-06.2007

Copyright $\odot 2007$ Society for Neuroscience $\quad$ 0270-6474/07/271346-10\$15.00/0 struct was solidified by demonstrating that burst-tonic/tonic neuron responses in the prepositus hypoglossi $(\mathrm{PH})$ and adjacent medial vestibular nuclei (VN) (collectively referred to here as $\mathrm{PH}-\mathrm{BT}$ ) correlate closely with eye position during static fixation and low-frequency slow eye movements (Baker and Berthoz, 1975; Lopez-Barneo et al., 1982; Escudero et al., 1992, 1996; McFarland and Fuchs, 1992). Consequently, PH-BT neurons were thought to encode the eye position component of the inverse dynamic model.

In recent years, however, at least two observations have challenged this theoretical construct. First, unlike rotation, the translational vestibulo-ocular reflex (TVOR) does not show clear evidence for processing by an inverse model. Specifically, the TVOR is robust only at high frequencies $(>0.5 \mathrm{~Hz})$ (Paige et al., 1991a; Telford et al., 1997; Angelaki, 1998). As a result, canceling the eye plant dynamics may be unnecessary in the TVOR, alleviating the need for processing otolith signals by an inverse model (Green and Galiana, 1998; Musallam and Tomlinson, 1999). Second, although PH-BT cell responses are more closely correlated with eye position than are those of other premotor cell types, they also carry signals related to eye velocity (McFarland and Fuchs, 1992). As an alternative, it was suggested that PH-BT neurons instead encode an estimate of the motor command (McCrea and Baker, 1985; Belknap and McCrea, 1988). However, PH-BT cell responses have never been compared with those of extraocular motoneurons over a broad frequency range sufficient to explicitly test this alternative hypothesis. These issues prompt a critical reevaluation of two conceptual ideas fundamental for understanding the neural control of slow eye movements: (1) the exis- 
tence of a dedicated inverse model for all eye movement types and (2) the nature of the internally estimated signal encoded by $\mathrm{PH}-\mathrm{BT}$ cells. In the current study, we reexamine these fundamental concepts by directly comparing $\mathrm{PH}-\mathrm{BT}$ responses with those of other premotor cell types and extraocular motoneurons, taking advantage of both the sensorimotor differences between the RVOR and TVOR and their shared high-frequency range of operation.

\section{Materials and Methods}

Animal preparation. Four juvenile Macacca mulatta and one Macacca fascicularis monkeys were prepared for chronic recording of binocular eye movements and single-unit activities. Each animal was chronically implanted with a delrin head stabilization ring that was secured to the skull with inverted stainless steel $\mathrm{T}$ bolts. A delrin recording platform (consisting of a staggered matrix of holes spaced $0.8 \mathrm{~mm}$ apart) was stereotaxically placed inside the ring and served as a guide for electrode placement. In three of the animals, the platform was implanted with a $10^{\circ}$ lateral/medial slant to allow bilateral access to the prepositus hypoglossi and abducens/oculomotor nerves and nuclei. Each animal was also implanted with dual eye coils on both eyes that were calibrated as explained in detail previously (Angelaki, 1998; Angelaki et al., 2000). All surgical procedures were performed under sterile conditions in accordance with institutional and National Institutes of Health guidelines.

Experimental set-up. During experiments, monkeys were seated upright in a primate chair secured inside a motion delivery system that consisted of a three-dimensional (3D) vestibular turntable mounted on a linear sled (Acutronics, Pittsburgh, PA). Binocular eye movements were measured with a three-field magnetic search coil system (16 inch cube; CNC Engineering, Seattle, WA) that was attached to the inner gimbal of the vestibular turntable. Visual targets were back-projected onto a flat screen mounted $20 \mathrm{~cm}$ away from the animal. A wall-mounted laser and $\mathrm{x}-\mathrm{y}$ mirror galvanometer system (General Scanning, Billerica, MA) provided world-fixed targets for gaze stabilization during head/body motion. A second laser-galvanometer system was mounted on top of the vestibular turntable such that it moved with the animal and provided a head-fixed target; this enabled evaluation of neural responses to vestibular stimulation when eye movements were suppressed or cancelled (i.e., VOR cancellation tasks). The second system was also used to provide visual targets for fixation and smooth pursuit tasks.

Stimulus presentation and data acquisition were controlled with custom-written scripts within the Spike2 software environment using the Cambridge Electronics Device (model power 1401; CED, Cambridge, England) data acquisition system. The voltage waveforms associated with each of the two eye-coil assemblies, velocity and/or position feedback signals from the rotator and linear sled and the signals from a 3D linear accelerometer that was mounted firmly to the inner gimbal of the rotator were stored on a computer for off-line analysis. All analog waveforms were anti-alias filtered $(200 \mathrm{~Hz}$, six-pole Bessel) and digitized at a rate of $833.33 \mathrm{~Hz}$ (CED model 1401, 16-bit resolution).

Extracellular single-unit responses were recorded using epoxy-coated, etched tungsten microelectrodes (FHC, Bowdoinham ME; Microprobe, Gaithersburg MD; custom-made) that were inserted into 26-gauge stainless-steel guide tubes (outside diameter of $457 \mu \mathrm{m}$ ), and then advanced through a predrilled hole in the recording platform. Neural activity was amplified, filtered ( $300 \mathrm{~Hz}$ to $6 \mathrm{kHz}$ ), and passed both to an audio amplifier and to a BAK Instruments dual time-amplitude window discriminator. Acceptance pulses from the window discriminator were used to trigger the event channel of the CED data acquisition system that stored the time of each spike at a $10 \mu$ s resolution. Neural waveforms, as well as all eye movement and stimulus signals, were also recorded using a digital audio tape drive (Cygnus Technologies, Delaware Water Gap, PA) to allow off-line cell reisolation.

Experimental protocols and neural recording. Animals were trained for juice rewards to fixate and/or track moving visual targets, as well as to maintain fixation of remembered target location for $1-2 \mathrm{~s}$ during motion in darkness. Behavioral performance under target viewing conditions was continuously monitored using both monocular $\left(1.5^{\circ}\right)$ and vergence
A

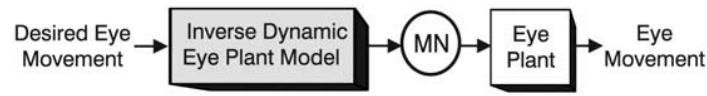

\section{B Parallel-Pathway}

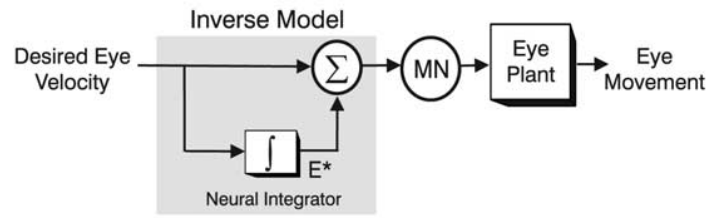

\section{Common Internal Model}

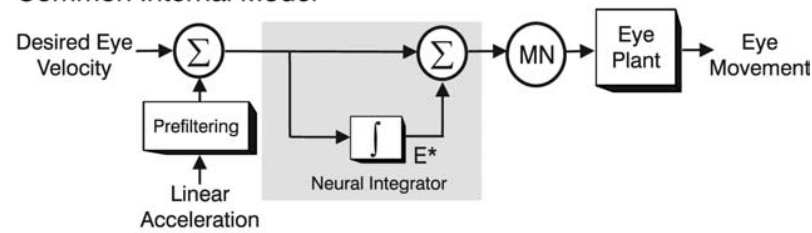

\section{Distributed Dynamic Processing}

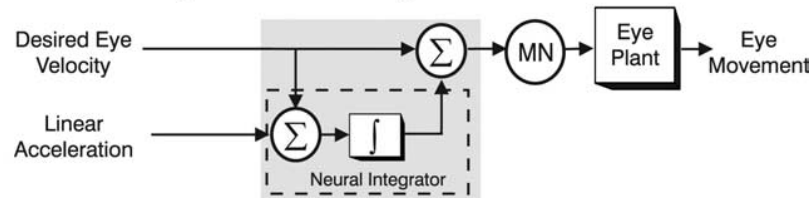

Figure 1. Schematic illustration of hypotheses for the dynamic processing underlying eye movement generation. $A$, Desired eye velocity commands are processed by an inverse dynamic model of the eye plant before being conveyed onto extraocular MNs. B, Parallel-pathway implementation of the inverse dynamic model. The inverse model (gray shaded box) is constructed by summing a weighted combination of desired eye velocity (top) and integrated eye velocity signals (bottom). An internal estimate of desired eye position ( $\left.E^{*}\right)$ is presumed to exist at the output of the neural integrator $\left(\int\right)$. C, Common internal model hypothesis for extending the framework to translation (TVOR). Otolith signals, encoding linear acceleration, are presumed to be "prefiltered" before converging on the inverse model used to convert desired eye velocity signals into appropriate motor commands (supplemental text, available at www.jneurosci.org as supplemental material). $\boldsymbol{D}$, Distributed dynamic processing hypothesis for the TVOR. The internal model is not fully implemented in the TVOR pathways. Otolith signals are presumed to be processed by only the neural integrator portion (dashed box) of the inverse model that converts linear acceleration signals into velocity. To explain the high-pass nature of the TVOR, it is assumed that the dynamic characteristics of the eye plant remain uncompensated and therefore contribute to shaping the reflex at the higher frequencies where the TVOR exhibits a robust response (see supplemental text, available at www.jneurosci.org as supplemental material).

$\left(1.5^{\circ}\right)$ windows (Angelaki et al., 2000; Meng et al., 2005). During motion in darkness (see below), animals were trained to maintain a verged eye position (within a $2-3^{\circ}$ vergence window) centered on the location of the previously viewed target.

In separate experiments, several brainstem areas were explored. In three animals, we recorded from burst-tonic neurons and other eye movement-sensitive cell types in the nucleus $\mathrm{PH}$ and adjacent medial VN [including the marginal zone, an area shown previously to project to the abducens and oculomotor nuclei (Langer et al., 1986)]. These recording locations extended bilaterally $0.5-2.4 \mathrm{~mm}$ caudal to the abducens nuclei, which were identified based on their characteristic burst-tonic activity (Fuchs and Luschei, 1970; Fuchs et al., 1988). We used several criteria to distinguish the prepositus region from the abducens nuclei, including (1) the presence of neurons with contralaterally directed eye movement preference intermingled with the more frequently encountered cells with ipsilateral on-directions, (2) the occasional presence of neurons with vertical eye movement preference and/or neurons whose firing rate modulated during motion in the absence of eye movement, (3) variable saccadic responsiveness, including cells that ranged from exhibiting clear bursts (i.e., burst-tonic neurons) to either weak or nonexistent burst activity (tonic 
neurons), and (4) the presence of neurons without a clear pause for off-direction saccades (McFarland and Fuchs, 1992; Kaneko, 1997).

Whenever an eye-movement-sensitive neuron was isolated, it was first classified based on its responses during horizontal/vertical smooth pursuit $\left(0.5 \mathrm{~Hz} \pm 12.4^{\circ}\right)$, visually guided saccades $\left( \pm 20^{\circ}\right)$, and yaw rotation $\left(0.5 \mathrm{~Hz}, \pm 10^{\circ}\right)$ while fixating a head-fixed target (RVOR cancellation). In particular, cells that exhibited clear responses for horizontal eye movements during all protocols but failed to respond to vestibular stimulation in the absence of eye movement were classified as burst-tonic/tonic cells and are collectively referred to here as PH-BT neurons. According to the classification of Scudder and Fuchs (1992), positionvestibular-pause (PVP) neurons exhibited responses to head rotation during RVOR cancellation and to eye movement during pursuit that were oppositely directed such that these signals superimposed in an additive manner (i.e., larger net response) during stabilization of a world-fixed target. Those with ipsilaterally directed head and contralaterally directed eye movement sensitivities were classified as type I PVP (PVP-I) whereas those with contralaterally directed head and ipsilaterally directed eye movement sensitivities were classified as type II PVP (PVP-II). Eye-head (EH) neurons exhibited responses to head motion during RVOR cancellation and to eye movement during smooth pursuit in the same direction, such that the two signals opposed each other during rotation while stabilizing a world-fixed target. EH cells included units with ipsilaterally directed eye and head velocity sensitivities (i-EH) as well as cells with contralaterally directed eye and head velocity sensitivities (c-EH). The majority of these neurons exhibited bursts during saccades in at least one direction. Many c-EH cells also exhibited a nonlinear encoding of horizontal eye position and/or small sensitivities to vertical eye movement in addition to horizontal eye movement during pursuit.

$\mathrm{PH}-\mathrm{BT}$ and other premotor cell types were then further tested during yaw rotations and lateral translations across a range of frequencies $(0.5-5$ $\mathrm{Hz})$. For translation, stimuli had a peak velocity of $\sim 14-16 \mathrm{~cm} / \mathrm{s}$. For rotation, the stimulus amplitudes at $0.5 \mathrm{~Hz}\left( \pm 10^{\circ}\right)$ and $1 \mathrm{~Hz}\left( \pm 5^{\circ}\right)$ were chosen to elicit similar eye velocities to those during pursuit/translation. At higher frequencies, stimulus amplitudes were constrained by the acceleration limit of the rotator $\left(220^{\circ} / \mathrm{s}^{2}\right)$. At each frequency, motion cycles were delivered with alternating light-dark periods in which monkeys were required to fixate on either a world-fixed target (light on) or its remembered location (light off). Other than a vergence window (see above), no other behavioral requirement was enforced during motion in darkness (because ocular responses to translation in the absence of visual feedback are typically undercompensatory) (Telford et al., 1997; Paige et al., 1998). Alternating light-dark periods were repeated until sufficient numbers of cycles were collected.

In addition to the premotor neurons, we also recorded motoneuron activities from two additional animals. This was done to enable a comparison of premotor neuron responses with those of extraocular motoneurons. Data were collected from 11 horizontal oculomotor nerve fibers and seven abducens neurons (three from the abducens nerve; four from the nuclei). The oculomotor nerve recordings were made rostral to the oculomotor nuclei [same locations/animals as used by Ghasia and Angelaki (2005)]. The majority of abducens recordings were made using the locations identified through electrical microstimulation [same locations/animals as used in Klier et al. (2006)]. In contrast to stimulation of the $\mathrm{PH}$ and $\mathrm{VN}$, abducens nerve/nucleus stimulation results in a transient deviation of only the ipsilateral eye [Klier et al. (2006), their Fig. 2]. Motoneuron responses were examined using the same sets of stimulus paradigms as those used for premotor cells.

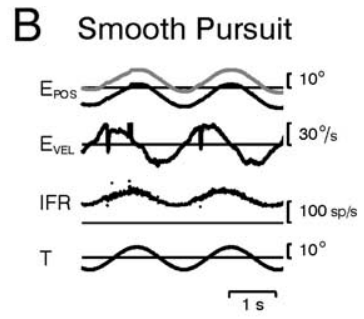

C RVOR Cancellation

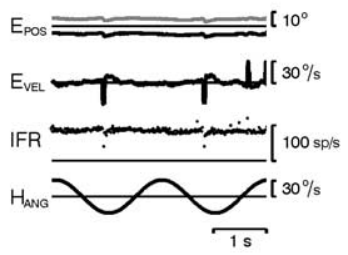

$4 \mathrm{~Hz}$ Space-Fixed
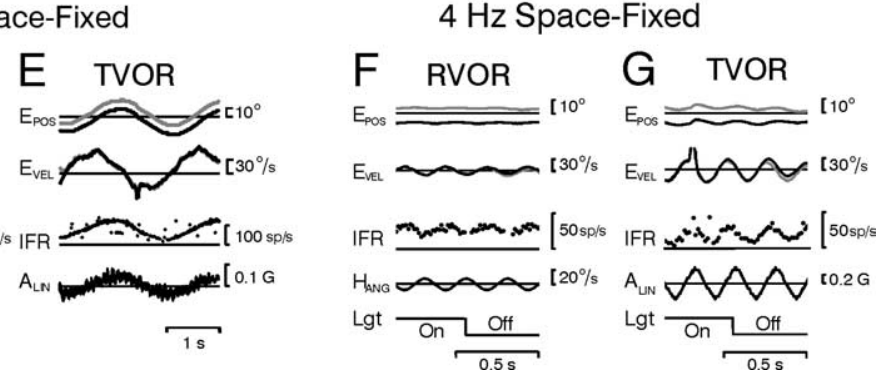

Figure 2. Horizontal burst-tonic cell recorded in the left prepositus hypoglossi. $\boldsymbol{A}-\boldsymbol{G}$, Responses during saccadic eye movements and fixations in darkness $(\boldsymbol{A})$, smooth target tracking $(\boldsymbol{B})$, rotation while stabilizing a head-fixed target (RVOR cancellation) $(\boldsymbol{C})$, rotation (RVOR) or translation (TVOR) at a frequency of $0.5 \mathrm{~Hz}$ while stabilizing a space-fixed target $(\boldsymbol{D}, \boldsymbol{E})$, and rotation or $\mathrm{E}_{\mathrm{POS}}$, Eye position (right eye, gray; left eye, black); $\mathrm{E}_{\mathrm{VEL}}$, eye velocity; $\mathrm{T}$, target position; $\mathrm{H}_{\mathrm{ANG}}$, angular head velocity; $\mathrm{A}_{\mathrm{LIN}}$, linear head acceleration; IFR, instantaneous firing rate; Lgt, lighting conditions (visual target on/off).

Data analyses. All data were analyzed off-line using Matlab (Mathworks, Natick, MA). Eye position was calibrated and expressed as threedimensional rotation vectors, as described in detail previously (Angelaki, 1998; Angelaki et al., 2000). Motion stimuli and eye movements were expressed in a right-handed coordinate frame such that positive horizontal plane angular and linear deviations were leftward and positive vertical angular deviations were downward. Saccades and fast phases of nystagmus were identified using a semiautomated computer algorithm based on a higher derivative of eye velocity. Automatic detection of saccades was followed by a manual inspection of the eye movements to correct any misidentifications (Angelaki, 1998). Fast eye movements and the corresponding firing activities during fast phases were removed from additional analysis. Note that the present analysis focuses exclusively on slow eye movements. PH-BT firing rate dynamics have been characterized previously during saccades and shown to be similar to those of abducens neurons (Sylvestre et al., 2003).

The instantaneous firing rate of a neuron's response was calculated as the reciprocal of interspike interval and assigned to the middle of the interval. Multiple cycles of data over time were overlaid. The dynamic characteristics (gain and phase) of each neuron were then computed by fitting a sine function (first and second harmonics and an offset) to the overlaid data using a nonlinear least-squares algorithm based on the Levenberg-Marquardt method. Under target viewing conditions, only portions of data in which the positions of both eyes were within $\pm 1^{\circ}$ of the target were included in the analyses. Under dark conditions, the portions of data selected for analysis were chosen so that at each frequency the data corresponded to approximately the same average vergence angle (within $\pm 1.25^{\circ}$ of the mean across all frequencies). Neural response gain and phase during motion were subsequently expressed relative to stimulus velocity, with gains in units of spikes/second/degree/ second (rotation) or spikes/second/centimeter/second (translation). In addition, neural responses during rotation, translation, and pursuit were also expressed relative to the evoked eye movement with gains in units of spikes/second/degree and phase expressed relative to eye position. Because data were typically recorded from both sides of the brainstem and cell on direction could be either ipsilateral or contralateral, phase values were expressed relative to the preferred eye or head movement direction of the cell to facilitate comparison of dynamic response properties.

Statistical comparisons were based on ANOVA using the SPSS statistics package (version 12; SPSS, Chicago, IL). Correlations between neural 
response gains and phases during rotations and translations were evaluated using linear regression. To account for the fact that the variables involved were independent of each other, slopes were calculated based on minimizations of the perpendicular offset of the data to the regression line using a custom-written Matlab script. Confidence intervals $(\mathrm{CI}$, 95\%) for the regressions were computed based on the distribution of slopes obtained when bootstrapping with replacement was performed on the data.

\section{Results}

\section{Theoretical basis for the tested hypotheses}

An implementation of the inverse dynamic model in the oculomotor pathways (Fig. 1A) was first proposed by Robinson et al. (Skavenski and Robinson, 1973; Robinson, 1981) who pioneered what has become well known as the parallel-pathway model (Fig. $1 B)$. Desired eye velocity signals were proposed to be conveyed to motoneurons (MNs) both directly and indirectly via a "neural integrator" (Fig. $1 B, \int$ ). Together, the two pathways compensate for the viscoelastic properties of the eyeball and are thought to comprise an inverse dynamic model of a simplified (first-order) eye plant. In alternative, but equivalent, representations the integration was implemented in a distributed manner either via positive feedback loops through a forward model of the eye plant (Galiana and Outerbridge, 1984; Galiana, 1991) (supplemental text, available at www.jneurosci.org as supplemental material) or more generally via recurrent connections in a dynamic neural network (Cannon and Robinson, 1985; Arnold and Robinson, 1991, 1997). Such distributed models have illustrated the ability to reproduce more realistic distributions of cell types that exhibit varying degrees of correlation with eye velocity versus position. Importantly, however, these implementations all predict the existence of neurons that either explicitly provide an internal estimate of eye position (Fig. $1 B, \mathrm{E}^{*}$ ) or a signal that is significantly more position-like than the signals encoded by extraocular motoneurons. PH-BT neurons have classically been assigned a dominant role in encoding this mainly "position-like" signal.

This theoretical framework has been questioned previously in the context of the eye movements elicited to compensate for translational motion (TVOR) (Green and Galiana, 1998; Musallam and Tomlinson, 1999). In particular, whereas the extended bandwidth of the RVOR provides direct evidence that the dominant eye plant dynamics have been compensated for neurally, responses to translation are robust only at high frequencies ( $>\sim 0.5-1 \mathrm{~Hz}$ ) (Paige et al., 1991a; Telford et al., 1997; Angelaki, 1998). Thus, in contrast to the case of the RVOR, the dynamic characteristics of the TVOR do not demonstrate evidence for processing by an inverse plant model. Neural processing in the TVOR is further complicated, however, by the fact that the information about linear motion provided by otolith afferents is encoded in terms of acceleration (Fernández and Goldberg, $1976 a, b)$. This is in contrast to the case of other types of eye movements including saccades, smooth target tracking, and the RVOR, where desired eye movement commands are encoded by predominantly velocity-like signals.

To explain how the TVOR fits into existing notions of the premotor processing underlying eye movement generation, two hypotheses have been proposed. The "common internal model" hypothesis suggests that a common inverse model is indeed shared for all eye movements, but evidence for processing by this model remains unobservable behaviorally during translation because otolith signals undergo additional prefiltering (Fig. 1C) (Paige and Tomko, 1991a,b; Telford et al., 1997). To a first approximation, this prefiltering network consists of an additional neural integrator to convert acceleration signals into velocity-like
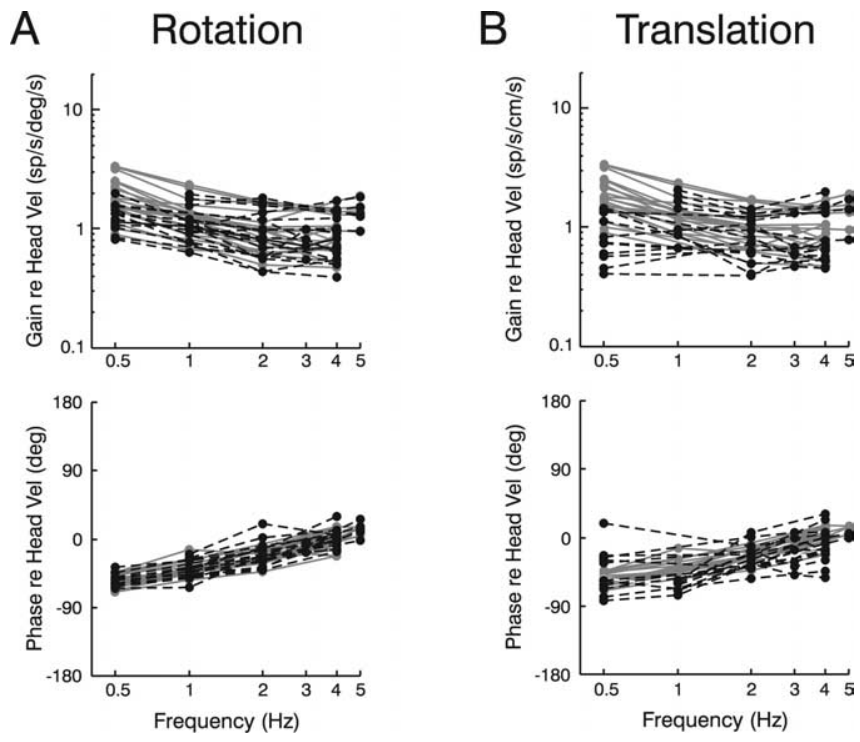

Figure 3. $\quad \boldsymbol{A}, \boldsymbol{B}$, Frequency response characteristics of PH-BT cells during rotation $(\boldsymbol{A})$ and translation $(\boldsymbol{B})$. Response gains and phases are expressed relative to head velocity during either rotation or translation both in the presence (gray curves) and absence (dashed black curves) of visual feedback (i.e., light and dark conditions, respectively). The majority of cells (86\%) responded to ipsilaterally directed eye or contralaterally directed head motion. Cell response phase has, thus, been expressed relative to contralaterally directed head velocity. Response phase in three cells that were excited for contralaterally directed eye or ipsilaterally directed head motion has been transposed by $180^{\circ}$ to facilitate comparison with the dynamic characteristics of the other cells.

commands and a high-pass filter to account for the high-pass nature of the TVOR. In contrast, the "distributed dynamic processing" hypothesis proposes that only a portion of the inverse model (the neural integrator) is implemented as part of the sensorimotor transformations during translation (Fig. 1D) (Green and Galiana, 1998; Musallam and Tomlinson, 1999; Angelaki et al., 2001). The integrative properties of the premotor circuitry (Fig. 1D, $\int$ pathway denoted by the dashed box) are used to convert otolith acceleration signals into velocity-like commands. However, instead of being processed by an inverse model, the eye plant remains uncompensated and provides the additional lowpass filtering required to ensure eye movement in phase with head movement at the higher frequencies where the TVOR exhibits a robust response (for additional details, see supplemental text, available at www.jneurosci.org as supplemental material).

The distributed dynamic processing hypothesis is computationally more efficient, as there is no need for additional neural circuitry to process sensory signals with different dynamic characteristics. However, a disadvantage of this scheme is that there may exist no consistent brainstem representation of the motor output. For example, in the scheme of Figure $1 D$, because otolith-derived acceleration signals are proposed to be temporally integrated at the premotor level only once, $\mathrm{PH}-\mathrm{BT}$ neuron responses would encode a signal more closely related to eye velocity during translation, but to eye position during rotation (Green and Galiana, 1998). In contrast, in the common internal model scheme, the final stage of premotor processing is shared for all types of eye movements, thus providing a consistent efferent copy signal during all eye movements.

Although both hypotheses can approximate the observed dynamics of the TVOR, each embeds fundamentally different predictions with respect to the properties of premotor neurons and their function. Thus, to distinguish between the two schemes of 
Figure 1, $C$ and $D$, three types of analyses will be presented. First, we investigate whether the same inverse model is used in the premotor processing of all slow eye movements. The common inverse model hypothesis predicts that, similar to motoneurons, $\mathrm{PH}-\mathrm{BT}$ neurons should exhibit the same relationship to the elicited eye movement during either rotation or translation, despite dynamically distinct sensory drives from semicircular canal and otolith afferents and dynamically distinct motor responses. Second, we directly compare $\mathrm{PH}-\mathrm{BT}$ responses with those of motoneurons to test whether PH-BT responses are more consistent with approximating an internal estimate of eye position $\left(\mathrm{E}^{\star}\right)$, as suggested by the theoretical representations in Figure $1, B$ and $C$, or alternatively, encode a consistent estimate of the motor command. Such a motor command estimate may serve the role of an "efference copy" signal when distributed via projections from the prepositus and vestibular nuclei to different brain areas beyond the extraocular motoneuron pools (e.g., cerebellum, superior colliculus, and thalamus) (McCrea and Baker, 1985; Belknap and McCrea, 1988; McCrea and Horn, 2005). Finally, we compare the dynamics of $\mathrm{PH}-\mathrm{BT}$ neurons with those of other premotor neurons to further establish whether PH-BT cells play a role that is unique among premotor cell types.

\section{General experimental observations}

We recorded neural responses from 25 horizontal PH-BT cells, 22 horizontal PVP cells (7 PVP-I; 15 PVP-II), 23 horizontal EH cells ( 8 i-EH; $15 \mathrm{c}-\mathrm{EH}$ ), and 18 horizontal motoneurons (11 oculomotor; 7 abducens). An example of a typical PH-BT neuron is illustrated in Figure 2. Consistent with the postulated role of this neuron type in coding an estimate of eye position (Fig. $1 B, C, \mathrm{E}^{\star}$ ), firing rate changed monotonically with deviations in eye position, exhibiting increased tonic activity for fixations to the left and decreased activity for fixations to the right (Fig. 2A). These were accompanied by bursts for leftward (i.e., on-direction) saccades and at least partial pauses for rightward saccades. Similarly, during low-frequency $(0.5 \mathrm{~Hz})$ smooth target tracking (Fig. $2 \mathrm{~B}$ ), rotation $(D)$ or translation $(E)$, the cell's firing rate closely followed the deviation in eye position. When eye movements were "suppressed" or "cancelled" during fixation of a target that moved with the head, the cell ceased to modulate (Fig. $2 C$ ). In contrast to these lower frequencies, however, at high frequencies (e.g., $4 \mathrm{~Hz}$ ) the cell modulated more closely in phase with eye velocity during both rotational and translational motion (Figs. $2 F, G$ ).

This observation is further emphasized in Figure 3, which summarizes how the entire population of PH-BT neurons responded to head motion across frequencies. To investigate their relationship to the sensory stimulus, response gains and phases have been expressed relative to head velocity during rotation/ translation in the presence (gray curves) and absence (dashed black curves) of visual feedback. Despite different afferent dynamics (otolith afferents encode linear acceleration; canal afferents encode angular velocity), PH-BT response dynamics appeared similar during rotation and translation. At high frequencies (e.g., 4-5 Hz) neurons modulated in phase with head velocity $\left(0^{\circ}\right.$ phase $)$ whereas at lower frequencies they exhibited
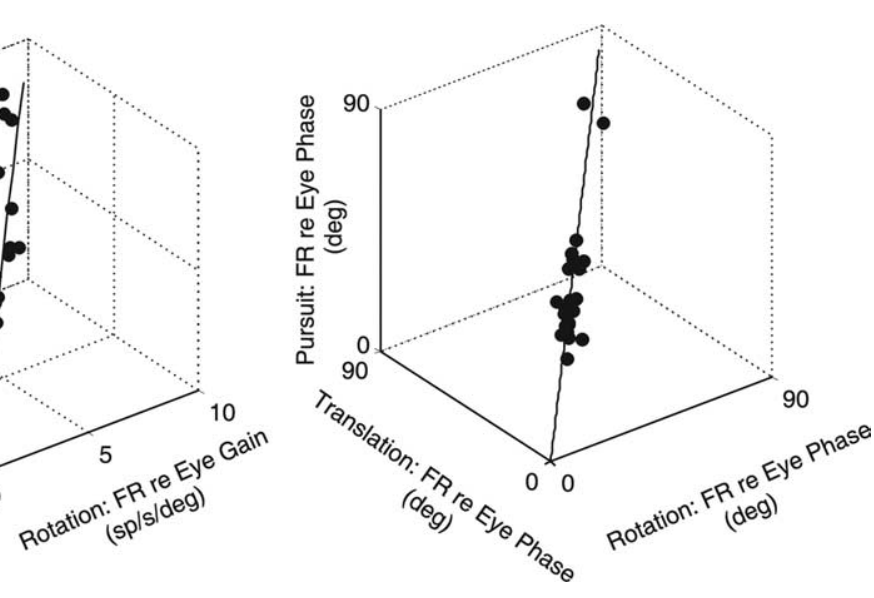

Figure 4. PH-BT responses during smooth pursuit, rotation, and translation $(0.5 \mathrm{~Hz})$. Gains (left) and phases (right) during smooth pursuit are compared with those during rotation or translation while stabilizing a space-fixed visual target. Gain refers to the neural firing rate modulation (FR, in spikes/second) relative to the evoked eye position (in degrees). Phase is expressed relative

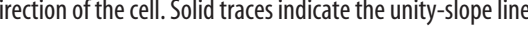

large phase lags. Notably, $0.5 \mathrm{~Hz}$ responses typically lagged head velocity by $40-70^{\circ}$ during both rotation (mean, $53^{\circ}$ in light; $55^{\circ}$ in dark) and translation (mean: $53^{\circ}$ in light; $52^{\circ}$ in dark). Because otolith afferents encode linear acceleration, these large phase lags during translation provide evidence for an additional integration of otolith as compared with canal signals, as suggested by the common internal model hypothesis (Fig. 1C). In contrast, these responses are inconsistent with the distributed dynamic processing strategy (Fig. 1D), because the single neural integrator in this scheme would have transformed the otolith acceleration signal into an estimate of head velocity but could not account for the additional phase lags observed at low frequencies.

\section{Common inverse dynamic model}

To directly evaluate whether $\mathrm{PH}-\mathrm{BT}$ neurons contribute to a common internal model, we next tested whether PH-BT neurons bore the same relationship to eye movement during voluntary pursuit and reflexive eye movements (RVOR/TVOR). Figure 4 illustrates neural response gains expressed relative to eye position. Data fell along the unity-slope line and gains were similar for all types of slow eye movement (means \pm SD, $5.0 \pm 1.9$ spikes/s/ deg for pursuit; $4.9 \pm 1.7$ spikes/s/deg for rotation; $5.4 \pm 1.8$ spikes/s/deg for translation). No difference was also seen for response phase (repeated measures ANOVA, $F_{(25,2)}=1.03 ; p>$ $0.05)$, which, in the majority of neurons, was close to but slightly leading eye position (means $\pm \mathrm{SD}, 35.4 \pm 12.7^{\circ}$ for pursuit; $36.5 \pm 12.4^{\circ}$ for rotation; $36.0 \pm 12.5^{\circ}$ for translation). Thus, in agreement with previous studies (McFarland and Fuchs, 1992), responses during $0.5 \mathrm{~Hz}$ visual and vestibular stimulation were compatible with the notion that these cells provide a consistent estimate of the motor output.

To investigate whether these observations hold more generally, we examined the relationship between $\mathrm{PH}-\mathrm{BT}$ responses and eye movement across different stimulus frequencies, comparing for the first time their response properties during rotation and translation at high frequencies both in the presence and absence of visual feedback. The neural gain and phase (expressed relative to eye position) during translation and rotation were significantly correlated (Fig. 5A), with population slopes across all stimulus frequencies that were indistinguishable from unity (light gain slope, 0.94; 95\% CI, 0.86-1.01; light phase slope, 0.96; 95\% CI, 0.93-0.99; dark gain slope, 0.99; 95\% CI, 0.90-1.09; dark phase 

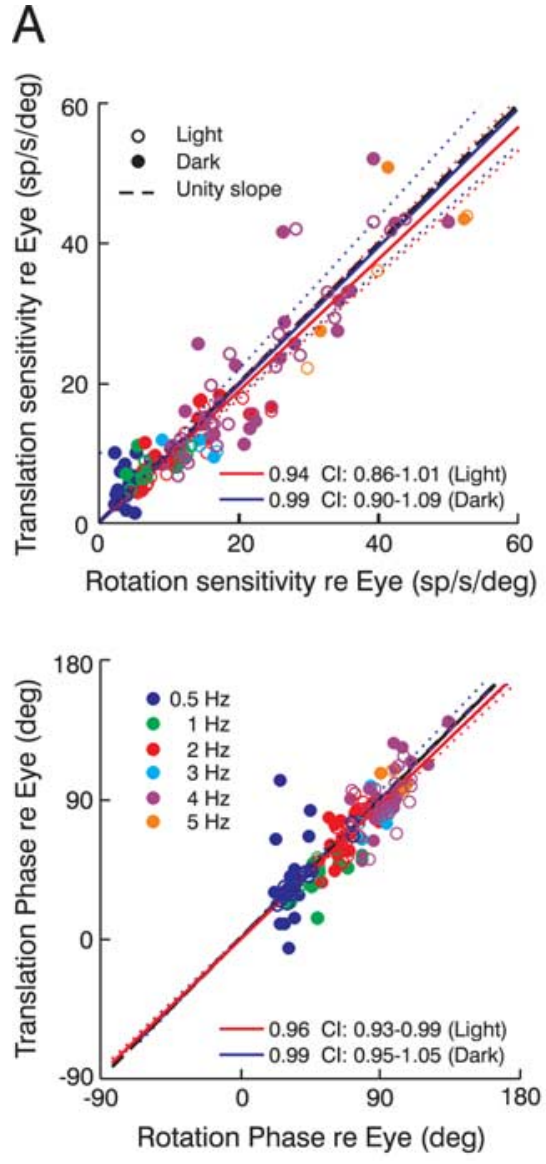

Figure 5. PH-BT responses during rotation and translation $(0.5-5 \mathrm{~Hz})$. $\boldsymbol{A}$, Neural response gains (top) and phases (bottom) relative to eye position during translation are compared with those during rotation. Data are plotted separately for different frequencies (blue, $0.5 \mathrm{~Hz}$; green, $1 \mathrm{~Hz}$; red, $2 \mathrm{~Hz}$; cyan, $3 \mathrm{~Hz}$; purple, $4 \mathrm{~Hz}$; orange, $5 \mathrm{~Hz}$ ) under either visual feedback (open circles) or dark (filled circles) conditions. Phases are expressed relative to the cell's preferred eye movement direction. $\boldsymbol{B}$, Mean translation/ rotation gain ratios (top) and translation-rotation phase differences (bottom) across frequencies under visual feedback conditions (gray bars) and in the dark (black bars). Error bars illustrate SD.

slope, $0.99 ; 95 \%$ CI, 0.95-1.05). When each frequency was considered separately, mean gain ratios [(translation re eye)/(rotation re eye)] were close to unity and phase differences [(translation re eye) - (rotation re eye)] were close to zero (Fig. $5 B$ ). Paired $t$ test comparisons of phase revealed no statistically significant differences at any frequency under visual feedback (light) conditions and most frequencies in the dark $(0.5,2,4,5 \mathrm{~Hz} ; p>$ $0.05)$. These results suggest that neural relationships to eye movement during rotation and translation were matched dynamically at the level of individual neurons. Similarly, no significant differences in gain were observed at any frequency during motion in darkness. Thus, PH-BT neurons in the prepositus hypoglossi and adjacent medial vestibular nuclei encode the same dynamic parameters of the evoked eye movement during rotations and translations. Notably, however, although neural responses bore a consistent relationship to the evoked eye movement, neural gain and phase were clearly dependent on stimulus frequency (Fig. 5A).

\section{Comparison between PH-BT and extraocular motoneuron dynamics}

The dependence of $\mathrm{PH}-\mathrm{BT}$ response gain and phase on frequency illustrates that, in contrast to a strict interpretation of the scheme in Figure $1 B$ (also see supplemental Fig. 1, available at www.jneurosci.org as supplemental material), PH-BT cells do not encode eye position. In a more liberal interpretation of the schemes of
Figure 1, however, it is often expected that PH-BT neurons should be closer to encoding eye position than extraocular motoneurons. Accordingly, PH-BT phases should lead eye position less than those of motoneurons. The failure of this prediction is illustrated in Figure 6, where the average gain and phase of $\mathrm{PH}-\mathrm{BT}$ neurons (triangles, solid lines) is compared with those of motoneurons (abducens: circles, dashed lines; oculomotor: squares, dotted lines). Each population demonstrated markedly similar behavior, characterized by increases in gain and phase leads with increasing frequency, such that at high frequencies (e.g., $4 \mathrm{~Hz}$ ), both premotor cells and motoneurons modulated more closely in phase with eye velocity $\left(90^{\circ}\right.$ phase) than eye position $\left(0^{\circ}\right.$ phase). $\mathrm{PH}-\mathrm{BT}$ gains and phases were indistinguishable from those of abducens neurons at any frequency during either rotation or translation (ANOVA, $p>0.05$ ). PH-BT cell responses were also statistically indistinguishable from oculomotor neuron responses in terms of both gain and phase during translation ( $p>0.05)$, but exhibited significantly larger phase leads relative to the motoneuron population during rotation (ANOVA, $p=0.002$ ). However, this small difference was actually in the opposite direction from that predicted by the classical models of Figure 1 ; PH-BT cells were actually further away from coding eye position (or closer to encoding eye velocity) than motoneurons. The observation that PH-BT cells were close to dynamically identical to extraocular motoneurons is consistent with the hypothesis that they encode an estimate of the motor command signal. Thus, these cells appear to represent the output (rather than merely an internal component) of the inverse dynamic model.

\section{Comparison between PH-BT and other premotor cell types}

To investigate whether PH-BT cells were unique in encoding a consistent stimulus-independent estimate of eye movement, we examined whether other premotor cell types bore the same relationship to the motor output during rotation and translation. This comparison, illustrated in Figure 7, is similar in format to that of Figure $5 A$ (top), but now includes data across all frequencies and lighting conditions from other premotor cell types (PVP-I, PVP-II, c-EH, and i-EH neurons). In contrast to PH-BT neurons, which demonstrated the same relationship to eye movement during both rotation and translation (Fig. 7, green), PVP and $\mathrm{EH}$ cells exhibited different sensitivities during rotation and translation. On average, the EH cell populations tended to exhibit larger sensitivities to the motor output during translation as compared with rotation (i-EH light slope, 1.65; 95\% CI, 1.05-2.02; i-EH dark slope, 1.86; CI, 1.17-2.21; c-EH light slope, 1.22; CI, 1.02-1.51; c-EH dark slope, 1.18; CI, 0.97-1.47). The converse was true for PVP cells (PVP-I light slope, 0.29; 95\% CI, 0.19- 
0.69; PVP-I dark slope, 0.27; 95\% CI, $0.17-$ 0.56; PVP-II light slope, $0.61 ; 95 \% \mathrm{CI}$, 0.41-0.89; PVP-II dark slope, 0.78; 95\% CI $0.58-0.96)$. Paired $t$ test comparisons across all frequencies and lighting conditions revealed that these gain differences were statistically significant $(p<0.05)$. Thus, $\mathrm{PH}-\mathrm{BT}$ firing rates were unique among all premotor cell types in exhibiting a consistent relationship to the motor output during rotations and translations.

Finally, to investigate whether PH-BT cells were also unique among premotor cell types in exhibiting response dynamics similar to those of motoneurons, Figure 8 illustrates the average phase differences between each premotor cell population and those of motoneurons at frequencies of $0.5,2$ and $4 \mathrm{~Hz}$. In contrast to PH-BT cells that exhibited close to zero mean phase differences relative to motoneurons, phase differences were much larger for all other cell types during rotation and for most cell types (i-EH, c-EH, PVP-II) during translation (ANOVA, $p<0.05$ ). Notably, not only PH-BT but also PVP-I cells exhibited similar phases to those of motoneurons during translation $(p>0.05)$. This latter observation, in conjunction with the small PVP-I gains during translation as compared with rotation (Fig. 7) (Meng and Angelaki, 2006) supports the notion that the signal flow for otolith and semicircular canal signals is at least partially different at the level of premotor neurons (for details, see Angelaki et al., 2001). Despite these differences, however, a consistent estimate of the motor output was constructed at the level of PH-BT cells. Furthermore, PH-BT cells were unique in this regard as they represented the sole cell population that not only encoded a stimulus-independent estimate of eye movement but also consistently exhibited motoneuron-like response dynamics.

\section{Discussion}

Using eye movements driven by different vestibular sensory stimuli across a range of frequencies $(0.5-5 \mathrm{~Hz})$, we have shown that $\mathrm{PH}-\mathrm{BT}$ neuron firing rates maintain a consistent relationship to the motor output regardless of the sensory stimulus driving the movement, supporting the notion of a common inverse model. We also identified the nature of this relationship by showing that their responses are dynamically similar to those of extraocular motoneurons. In contrast, none of the other premotor cell types exhibited a consistent relationship to the motor output during rotation and translation. We conclude that PH-BT cells play a unique role among premotor cell types in representing the output (rather than a component) of the inverse model and in providing a reliable copy of the motoneural command that may be distributed to other brain areas as an efference copy signal.

\section{Evidence for a common inverse dynamic model}

The notion of a shared integrative network has been well established by deficits in multiple eye movement types after damage (Cannon and Robinson, 1987; Cheron and Godaux, 1987; Gold-
Rotation

B Translation
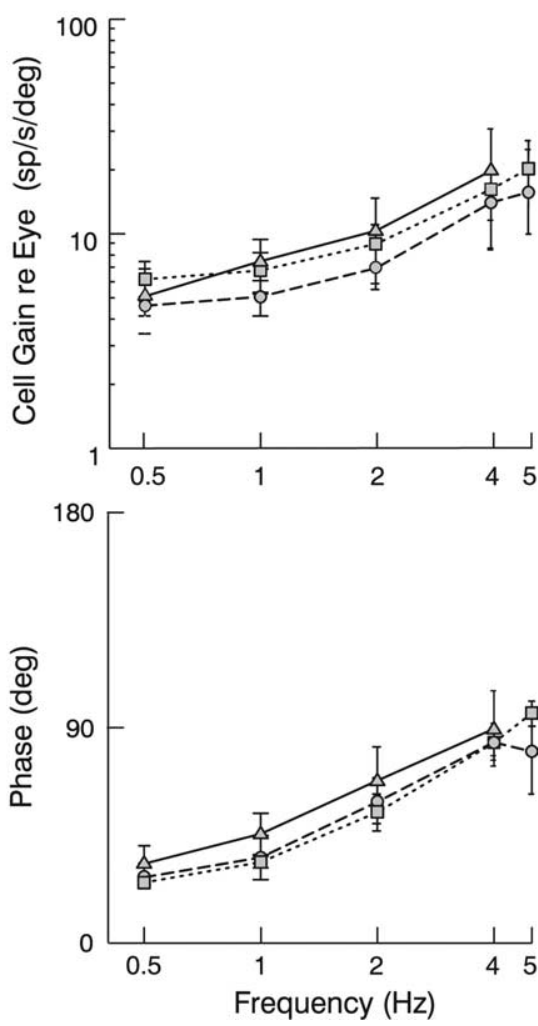


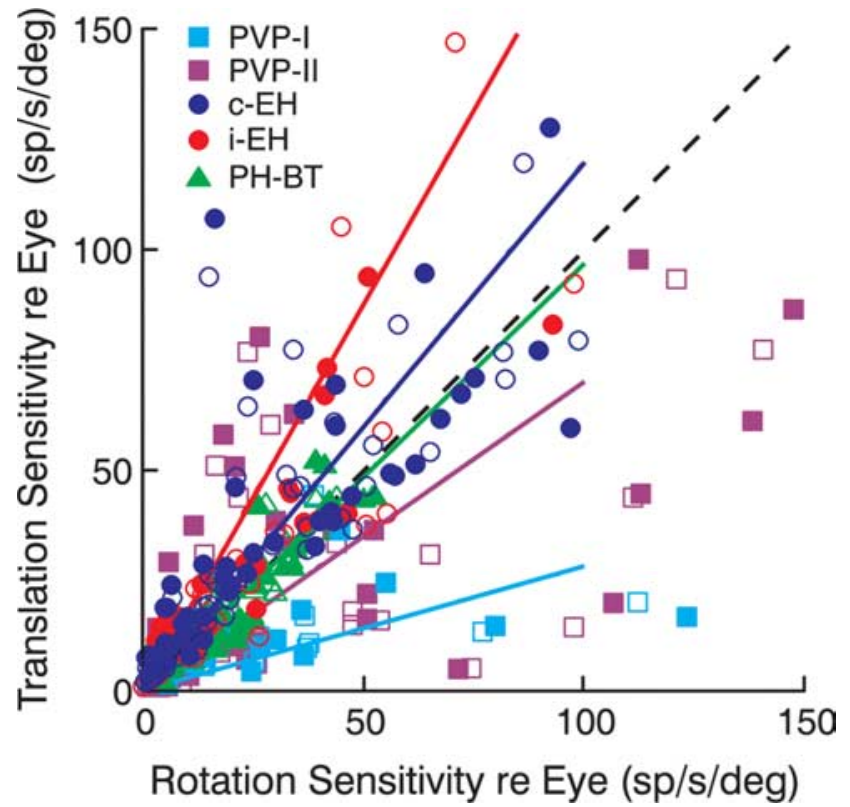

Figure 7. Comparison of premotor cell responses during rotation and translation. Neural response gains relative to eye position across all frequencies during translation are compared with those during rotation under visual feedback (open symbols) and dark conditions (filled symbols). Different symbols and colors denote different cell types (cyan squares, PVP-I; purple squares, PVP-II; blue circles, c-EH; red circles, i-EH; green triangles, PH-BT). Solid lines indicate the regression slopes for data across all frequencies and lighting conditions (PVP-I: $0.28,95 \% \mathrm{Cl}$, 0.20 - 0.48; PVP-II: $0.70,95 \% \mathrm{Cl}, 0.53-0.84 ;$ c-EH: $1.20,95 \% \mathrm{Cl}, 1.06-1.39 ; \mathrm{i}-\mathrm{EH}: 1.75,95 \% \mathrm{Cl}$, $1.28-2.04 ;$ PH-BT: $0.97,95 \% \mathrm{Cl}, 0.91-1.03)$. Dashed black line shows the unity slope.

common inverse model via partially distinct pathways from those of canal signals, as suggested previously (Angelaki et al., 2001) and supported by our current results (Figs. 7, 8).

\section{Role of PH-BT neurons in the neural implementation of an} inverse dynamic model

Despite a continuing prevalence of models like those in Figure 1 that emphasize the concept of a neural coding of eye position, numerous studies have shown that a consistent internal estimate of $\mathrm{E}^{\star}$ might not be coded explicitly by any premotor cell type (Baker and Berthoz, 1975; Lopez-Barneo et al., 1982; DelgadoGarcia et al., 1989; Escudero et al., 1992, 1996; McFarland and Fuchs, 1992; Scudder and Fuchs, 1992; Cullen et al., 1993; Sylvestre et al., 2003). This inconsistency between neurophysiological findings and the predictions of conceptual models (Fig. 1) has led to uncertainty over the role and significance of different premotor cell types in oculomotor function (McCrea and Baker, 1985; Belknap and McCrea, 1988; McFarland and Fuchs, 1992; Scudder and Fuchs, 1992; Lisberger et al., 1994). As a compromise between the schemes of Figure 1 and the observed presence of combined velocity/position signals in PH-BT cell firing rates, it is often assumed that PH-BT neurons carry more position-like signals than those of extraocular motoneurons. By examining the high-frequency responses of PH-BT neurons and by comparing them directly to those of motoneurons, we now show conclusively that this is not the case; their properties during slow eye movements are in fact identical to those of extraocular motoneurons.

PH-BT neurons do not therefore simply represent a portion of the inverse model circuitry, either as a dedicated eye position pathway (as the simplified schemes in Fig. 1 suggest) or as some combination of position/velocity signals that is more positionlike than motoneuron responses. Instead, they represent the out-
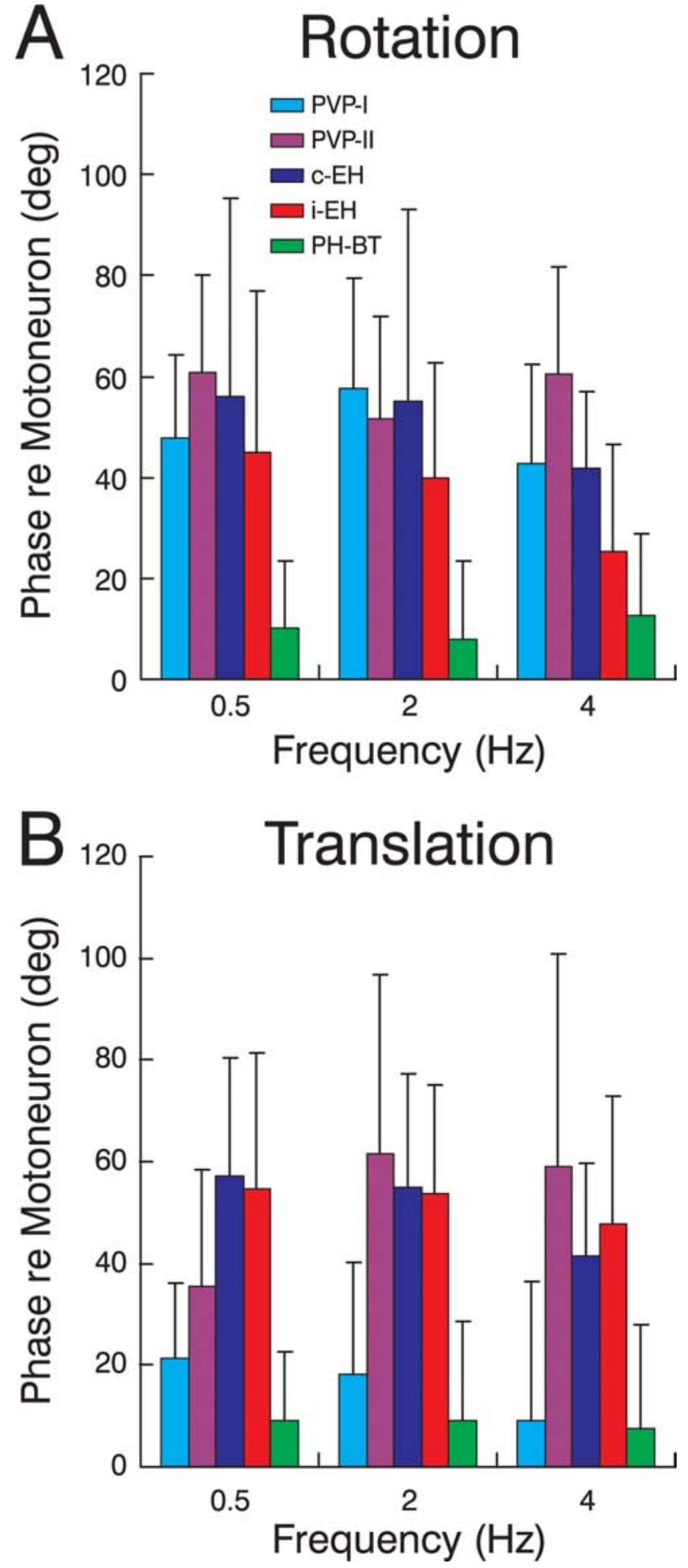

Figure 8. Comparison of the dynamic characteristics of different premotor cell types with those of extraocular motoneurons. $\boldsymbol{A}, \boldsymbol{B}$, The difference between the response phase of each cell type and the average motoneural phase (i.e., mean of $A B$ and $0 M$ cells) during rotation $(A)$ and translation (B) is illustrated for frequencies of $0.5,2$, and $4 \mathrm{~Hz}$ (cyan, PVP-l; purple, PVP-II; blue, (-EH; red, i-EH; green, PH-BT). Positive values indicate a phase lead relative to the average motoneural phase. Error bars illustrate SD.

put of the inverse model itself. This conclusion is consistent with neuroanatomical findings showing that all regions projecting to the abducens nuclei also project to the $\mathrm{PH}$ (Belknap and McCrea, 1988). However, it is unlikely that the primary role of PH-BT cells 


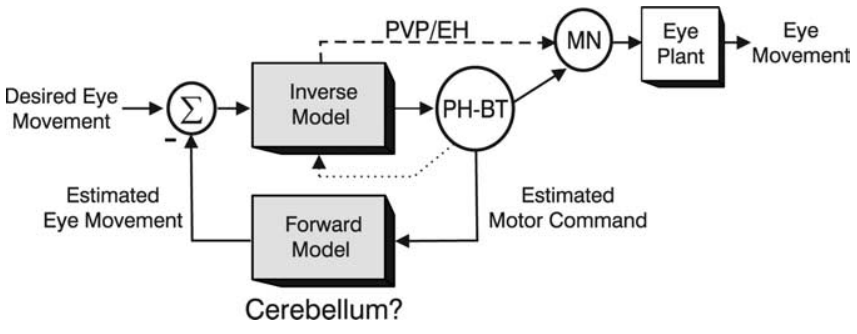

Figure 9. Schematic illustration of the dynamic processing for eye movement generation in which PH-BT cells represent the output of the inverse dynamic model. Although the PH-BT cell population is illustrated outside the box labeled "inverse model" for simplicity, these cells can also be considered to contribute as an integral part of the model through feedback interconnections (dotted line) with other premotor neural populations including PVP and EH neurons. PVP and EH cells, which may also participate in the inverse dynamic computations and are known to contribute to the motoneural command signal, are also shown projecting to the motoneuron population, MN. PH-BT cells are postulated to distribute an estimate of the motor command signal to other brain areas (e.g., the cerebellar flocculus) that potentially implement a forward model of the eye plant. The estimated motor response at the output of such a forward model can be compared with the desired eye movement to help refine the motor command signal.

is to provide the main source of premotor drive to motoneurons. Such a notion would be incompatible with anatomical and physiological evidence that the abducens nuclei receive potentially more extensive projections from other cell populations in the ventrolateral and medial vestibular nuclei (PVP and EH cells) (McCrea et al., 1987; Scudder and Fuchs, 1992) and reticular neurons (Strassman et al., 1986a,b; Scudder et al., 1988).

Functional utility of an efference copy of the motor command If the role of PH-BT cells is not simply to supply the appropriate drive to motoneurons, why do they carry a replica of the motoneural command? Because motoneurons are restricted to innervating the extraocular muscles, a primary function of $\mathrm{PH}-\mathrm{BT}$ cells must be to distribute a copy of the motor command (i.e., an efference copy) elsewhere in the brain (McCrea and Baker, 1985; Belknap and McCrea, 1988; McCrea and Horn, 2005). In particular, because the motor command signal reflects a mixed representation of estimated eye kinematics, it embeds essential information about both ongoing eye movement (e.g., eye velocity) and average (static) eye position. We therefore suggest that the signals carried by PH-BT neurons are used in at least two important ways: (1) during fixation they provide the brain with an estimate of current eye position, and (2) during movement an estimate of the motor command, constructed at the output of an inverse model, could be fed back through a forward model to compute the predicted consequences of this command (i.e., "estimated eye velocity") (Fig. 9) (see also Glasauer, 2003). This estimated eye velocity signal may be used to update the brain about ongoing eye movement. In addition, the error between predicted and desired action may be used to help refine the motor command. This latter concept, illustrated in Figure 9, stems from contemporary theories of limb motor control suggesting that the sensorimotor processing may involve the implementation of complementary forward and inverse models of the sensors and motor actuators (Flanagan and Wing, 1997; Wolpert and Kawato, 1998; Kawato, 1999). The cerebellum has been implicated in the implementation of such forward and inverse dynamic models for both limb control (Ito, 1970; Miall et al., 1993; Wolpert and Kawato, 1998; Kawato et al., 2003) and eye movements (Shidara et al., 1993; Gomi et al., 1998; Glasauer, 2003).

This notion is supported by the fact that $\mathrm{PH} / \mathrm{VN}$ neurons, as well as the paramedian tract cell group (Buttner-Ennever et al.,
1989; Nakamagoe et al., 2000), are known to project to the flocculus (Langer et al., 1985a; McCrea and Baker, 1985; Belknap and McCrea, 1988). Feedback from a presumed forward model in the flocculus to brainstem networks involved in constructing an inverse model may be provided via Purkinje cell projections onto floccular target neurons in the VN (Langer et al., 1985b; Lisberger et al., 1994). Furthermore, although the inverse model for eye movements, and in particular its output (PH-BT cells), could reside largely in the brainstem, feedback loops through the cerebellum are also likely to play a role in its implementation because the floccular loop contributes to the observed neural integrator dynamics (Zee et al., 1981).

In summary, we have shown that, in agreement with classical theoretical concepts, a common inverse model holds across eye movement types regardless of the dynamic characteristics of both the motor output and the underlying sensory drive. However, this occurs at the cost of additional neural processing for the TVOR. Importantly, the consistent internal estimate associated with this model is not of eye position, but instead of the motoneural command. This signal may provide the input to a forward model (postulated to at least partly exist in the cerebellum) (Fig. 9) (see also Glasauer, 2003) that is used to construct an internal prediction of the action to be performed ["reafference" principle (von Holst and Mittelstädt, 1950)]. The error between predicted and desired movement can subsequently be computed and used in a feedback loop to enhance (or adapt) the motor command. Although this organization merely presents a hypothesis at present, it provides a functional alternative to more traditional concepts of premotor oculomotor system organization. We suggest that classical interpretations of the premotor oculomotor circuitry should be updated to reflect a primary role for PH-BT neurons, not as the output of a neural integrator, but as the output of the inverse model that distributes an efference copy of the motoneural command.

\section{References}

Angelaki DE (1998) Three-dimensional organization of otolith-ocular reflexes in rhesus monkeys. III. Responses to translation. J Neurophysiol 80:680-695.

Angelaki DE, McHenry MQ, Hess BJ (2000) Primate translational vestibuloocular reflexes. I. High-frequency dynamics and three-dimensional properties during lateral motion. J Neurophysiol 83:1637-1647.

Angelaki DE, Green AM, Dickman JD (2001) Differential sensorimotor processing of vestibulo-ocular signals during rotation and translation. J Neurosci 21:3968-3985.

Arnold DB, Robinson DA (1991) A learning network model of the neural integrator of the oculomotor system. Biol Cybern 64:447-454.

Arnold DB, Robinson DA (1997) The oculomotor integrator: testing of a neural network model. Exp Brain Res 113:57-74.

Baker R, Berthoz A (1975) Is the prepositus hypoglossi nucleus the source of another vestibulo-ocular pathway? Brain Res 86:121-127.

Belknap DB, McCrea RA (1988) Anatomical connections of the prepositus and abducens nuclei in the squirrel monkey. J Comp Neurol 268:13-28.

Buttner-Ennever JA, Horn AK, Schmidtke K (1989) Cell groups of the medial longitudinal fasciculus and paramedian tracts. Rev Neurol (Paris) 145:533-539.

Cannon SC, Robinson DA (1985) An improved neural-network model for the neural integrator of the oculomotor system: more realistic neuron behavior. Biol Cybern 53:93-108.

Cannon SC, Robinson DA (1987) Loss of the neural integrator of the oculomotor system from brain stem lesions in monkey. J Neurophysiol 57:1383-1409.

Cheron G, Godaux E (1987) Disabling of the oculomotor integrator by kainic acid injections in the prepositus-vestibular complex of the cat. J Physiol (Lond) 394:267-290.

Cullen KE, Chen-Huang C, McCrea RA (1993) Firing behaviour of brain stem 
neurons during voluntary cancellation of the horizontal vestibuloocular reflex. II. Eye movement related neurons. J Neurophysiol 70:844-856.

Delgado-Garcia JM, Vidal PP, Gomez C, Berthoz A (1989) A neurophysiological study of prepositus hypoglossi neurons projecting to oculomotor and preoculomotor nuclei in the alert cat. Neuroscience 29:291-307.

Escudero M, de la Cruz RR, Delgado-Garcia JM (1992) A physiological study of vestibular and prepositus hypoglossi neurones projecting to the abducens nucleus in the alert cat. J Physiol (Lond) 458:539-560.

Escudero M, Cheron G, Godaux E (1996) Discharge properties of brain stem neurons projecting to the flocculus in the alert cat. II. Prepositus hypoglossal nucleus. J Neurophysiol 76:1775-1785.

Fernández C, Goldberg JM (1976a) Physiology of peripheral neurons innervating otolith organs of the squirrel monkey. I. Response to static tilts and to long-duration centrifugal force. J Neurophysiol 39:970-984.

Fernández C, Goldberg JM (1976b) Physiology of peripheral neurons innervating otolith organs of the squirrel monkey. III. Response dynamics. J Neurophysiol 39:996-1008.

Flanagan JR, Wing AM (1997) The role of internal models in motion planning and control: evidence from grip force adjustments during movements of hand-held loads. J Neurosci 17:1519-1528.

Fuchs AF, Luschei ES (1970) Firing patterns of abducens neurons of alert monkeys in relationship to horizontal eye movement. J Neurophysiol 33:382-392.

Fuchs AF, Scudder CA, Kaneko CR (1988) Discharge patterns and recruitment order of identified motoneurons and internuclear neurons in the monkey abducens nucleus. J Neurophysiol 60:1874-1895.

Galiana HL (1991) A nystagmus strategy to linearize the vestibulo-ocular reflex. IEEE Trans Biomed Eng 38:532-543.

Galiana HL, Outerbridge JS (1984) A bilateral model for central neural pathways in the vestibuloocular reflex. J Neurophysiol 51:210-241.

Ghasia FF, Angelaki DE (2005) Do motoneurons encode the noncommutativity of ocular rotations? Neuron 47:281-293.

Glasauer S (2003) Cerebellar contribution to saccades and gaze holding: a modeling approach. Ann NY Acad Sci 1004:206-219.

Goldman MS, Kaneko CRS, Major G, Aksay E, Tank DW, Seung HS (2002) Linear regression of eye velocity on eye position and head velocity suggests a common oculomotor neural integrator. J Neurophysiol 88:659-665.

Gomi H, Shidara M, Takemura A, Inoue Y, Kawano K, Kawato M (1998) Temporal firing patterns of Purkinje cells in the cerebellar ventral paraflocculus during ocular following responses in monkeys. I. Simple spikes. J Neurophysiol 80:818-831.

Green AM, Angelaki DE (2003) Resolution of sensory ambiguities for gaze stabilization requires a second neural integrator. J Neurosci 23:9265-9275.

Green AM, Angelaki DE (2004) An integrative neural network for detecting inertial motion and head orientation. J Neurophysiol 92:905-925.

Green AM, Galiana HL (1998) Hypothesis for shared central processing of canal and otolith signals. J Neurophysiol 80:2222-2228.

Ito M (1970) Neurophysiological aspects of the cerebellar motor control system. Int J Neurol 7:162-176.

Kaneko CR (1997) Eye movement deficits after ibotenic acid lesions of the nucleus prepositus hypoglossi in monkeys. I. Saccades and fixation. J Neurophysiol 78:1753-1768.

Kawato M (1999) Internal models for motor control and trajectory planning. Curr Opin Neurobiol 9:718-727.

Kawato M, Kuroda T, Imamizu H, Nakano E, Miyauchi S, Yoshioka T (2003) Internal forward models in the cerebellum: fMRI study on grip force and load force coupling. Prog Brain Res 142:171-188.

Klier EM, Meng H, Angelaki DE (2006) Three-dimensional kinematics at the level of the oculomotor plant. J Neurosci 26:2732-2737.

Langer T, Fuchs AF, Scudder CA, Chubb MC (1985a) Afferents to the flocculus in the cerebellum of the rhesus macaque as revealed by retrograde transport of horseradish peroxidase. J Comp Neurol 235:1-25.

Langer T, Fuchs AF, Chubb MC, Scudder CA, Lisberger SG (1985b) Floccular efferents in the rhesus macaque as revealed by autoradiography and horseradish peroxidase. J Comp Neurol 235:26-37.

Langer T, Kaneko CR, Scudder CA, Fuchs AF (1986) Afferents to the abducens nucleus in the monkey and cat. J Comp Neurol 245:379-400.

Lisberger SG, Pavelko TA, Broussard DM (1994) Responses during eye movements of brain stem neurons that receive monosynaptic inhibition from the flocculus and ventral paraflocculus in monkeys. J Neurophysiol 72:909-927.
Lopez-Barneo J, Darlot C, Berthoz A, Baker R (1982) Neuronal activity in prepositus nucleus correlated with eye movement in the alert cat. J Neurophysiol 47:329-352.

McCrea RA, Baker R (1985) Anatomical connections of the nucleus prepositus of the cat. J Comp Neurol 237:377-407.

McCrea RA, Horn AK (2005) Nucleus prepositus. Prog Brain Res 151:205-230.

McCrea RA, Strassman A, May E, Highstein SM (1987) Anatomical and physiological characteristics of vestibular neurons mediating the horizontal vestibulo-ocular reflex of the squirrel monkey. J Comp Neurol 264:547-570.

McFarland JL, Fuchs AF (1992) Discharge patterns in nucleus prepositus hypoglossi and adjacent medial vestibular nucleus during horizontal eye movement in behaving macaques. J Neurophysiol 68:319-332.

Meng H, Angelaki DE (2006) Neural correlates of the dependence of compensatory eye movements during translation on target distance and eccentricity. J Neurophysiol 95:2530-2540.

Meng H, Green AM, Dickman JD, Angelaki DE (2005) Pursuit-vestibular interactions in brain stem neurons during rotation and translation. J Neurophysiol 93:3418-3433.

Miall RC, Wolpert DM (1996) Forward models for physiological motor control. Neural Netw 9:1265-1279.

Miall RC, Weir DJ, Wolpert DM, Stein JF (1993) Is the cerebellum a Smith predictor? J Mot Behav 25:203-216.

Musallam WS, Tomlinson RD (1999) Model for the translational vestibuloocular reflex (VOR). J Neurophysiol 82:2010-2014.

Nakamagoe K, Iwamoto Y, Yoshida K (2000) Evidence for brainstem structures participating in oculomotor integration. Science 288:857-859.

Paige GD, Tomko DL (1991a) Eye movement responses to linear head motion in the squirrel monkey. I. Basic characteristics. J Neurophysiol 65:1170-1182.

Paige GD, Tomko DL (1991b) Eye movement responses to linear head motion in the squirrel monkey. II. Visual-vestibular interactions and kinematic considerations. J Neurophysiol 65:1183-1196.

Paige GD, Telford L, Seidman SH, Barnes GR (1998) Human vestibuloocular reflex and its interactions with vision and fixation distance during linear and angular head movement. J Neurophysiol 80:2391-2404.

Robinson DA (1981) The use of control systems analysis in the neurophysiology of eye movements. Annu Rev Neurosci 4:463-503.

Scudder CA, Fuchs AF (1992) Physiological and behavioral identification of vestibular nucleus neurons mediating the horizontal vestibuloocular reflex in trained rhesus monkeys. J Neurophysiol 68:244-264.

Scudder CA, Fuchs AF, Langer TP (1988) Characteristics and functional identification of saccadic inhibitory burst neurons in the alert monkey. J Neurophysiol 59:1430-1454.

Shadmehr R, Mussa-Ivaldi FA (1994) Adaptive representation of dynamics during learning of a motor task. J Neurosci 14:3208-3224.

Shidara M, Kawano K, Gomi H, Kawato M (1993) Inverse-dynamics model eye movement control by Purkinje cells in the cerebellum. Nature 365:50-52.

Skavenski AA, Robinson DA (1973) Role of abducens neurons in the vestibuloocular reflex. J Neurophysiol 36:724-738.

Strassman A, Highstein SM, McCrea RA (1986a) Anatomy and physiology of saccadic burst neurons in the alert squirrel monkey. I. Excitatory burst neurons. J Comp Neurol 249:337-357.

Strassman A, Highstein SM, McCrea RA (1986b) Anatomy and physiology of saccadic burst neurons in the alert squirrel monkey. II. Inhibitory burst neurons. J Comp Neurol 249:358-380.

Sylvestre PA, Choi JTL, Cullen KE (2003) Discharge dynamics of oculomotor neural integrator neurons during conjugate and disjunctive saccades and fixation. J Neurophysiol 90:739-754.

Telford L, Seidman SH, Paige GD (1997) Dynamics of squirrel monkey linear vestibuloocular reflex and interactions with fixation distance. J Neurophysiol 78:1775-1790.

von Holst E, Mittelstädt H (1950) Das Reafferenzprinzip (Wechselwirkung zwischen zentralnervensystem und peripherie). Naturwissenschaften $37: 464-476$

Wolpert DM, Kawato M (1998) Multiple paired forward and inverse models for motor control. Neural Netw 11:1317-1329.

Zee DS, Yamazaki A, Butler PH, Gucer G (1981) Effects of ablation of flocculus and paraflocculus of eye movements in primate. J Neurophysiol $46: 878-899$ 